\title{
Nonlinear vector susy for the three-dimensional topological massive Yang-Mills theory
}

\author{
C.A.G. Sasaki, \\ UERJ, Universidade Estadual do Rio de Janeiro; \\ Departamento de Estruturas Matemáticas; \\ IME, Instituto de Matemática; \\ Rua São Francisco Xavier 524, 20550-013, \\ Maracanã, Rio de Janeiro, Brazil. \\ D.G.G. Sasaki \\ CBPF, Centro Brasileiro de Pesquisas Físicas, \\ DCP, Departamento de Campos e Partículas. \\ Rua Dr Xavier Sigaud 150, 22290-180 Urca, \\ Rio de Janeiro, Brazil. \\ and \\ S.P. Sorella \\ UERJ, Universidade Estadual do Rio de Janeiro, \\ Departamento de Física Teórica; \\ IF, Instituto de Física; \\ Rua São Francisco Xavier 524, 20550-013; \\ Maracanã, Rio de Janeiro, Brazil. \\ UERJ/DFT-04/98 PACS: 11.10.Gh
}

February 14, 2018 


\begin{abstract}
A nonlinear vector supersymmetry for three-dimensional topological massive Yang-Mills is obtained by making use of a nonlinear but local and covariant redefinition of the gauge field.
\end{abstract}

\title{
1 Introduction
}

Nowadays, it is well established that the topological field theories (TFT) [1] are characterized, besides their BRST invariance, by a further symmetry carrying a Lorentz index [2, 3, 田, 5]. This additional invariance has been called vector supersymmetry since the corresponding generators give rise, together with the BRST generator, to a supersymmetric algebra of the Wess-Zumino type. The existence of the vector supersymmetry has been first detected in the case of the three-dimensional topological Chern-Simons theory quantized in the Landau gauge [2, 3] and later on has been extended to others TFT as, for instance, the BF models [4, [6] and the Witten's cohomological field theories [5]. It is worth remarking here that, actually, the existence of the vector susy can be established by purely cohomological methods, relying on the fact that the energy-momentum tensor of the TFT can be set in the form of an exact BRST variation [7].

On the other side, in a recent series of works [8, 9], it has been proven that three-dimensional gauge theories of the Yang-Mills type in presence of the topological Chern-Simons term can be cast in the form of a pure ChernSimons action through a nonlinear but local and covariant redefinition of gauge field. Indeed, in the case of the topological massive Yang-Mills theory whose action is given by the sum of the Chern-Simons action and of the Yang-Mills term

$$
S_{T M Y M}(A)=S_{C S}(A)+S_{Y M}(A),
$$

where

$$
\begin{gathered}
S_{Y M}(A)=\frac{1}{4 m} \operatorname{tr} \int d^{3} x F_{\mu \nu} F^{\mu \nu} \\
S_{C S}(A)=\frac{1}{2} \operatorname{tr} \int d^{3} x \varepsilon^{\mu \nu \rho}\left(A_{\mu} \partial_{v} A_{\rho}+\frac{2}{3} g A_{\mu} A_{\nu} A_{\rho}\right),
\end{gathered}
$$

we have

$$
S_{T M Y M}(A)=S_{C S}(\widehat{A}),
$$


with

$$
\widehat{A}_{\mu}=A_{\mu}+\sum_{n=1}^{\infty} \frac{1}{m^{n}} \vartheta_{\mu}^{n}(D, F) .
$$

The parameters $g, m$ in eqs.(2), (3) identify respectively the coupling constant and the so called topological mass [10]. As shown in ref. [8], the coefficients $\vartheta_{\mu}^{n}$ turn out to be local and covariant, meaning that they can be expressed in terms of the field strength $F_{\mu v}$ and its covariant derivatives. For instance, the first terms of the redefinition (5) have been found

$$
\begin{aligned}
\vartheta_{\mu}^{1}= & \frac{1}{4} \varepsilon_{\mu \sigma \tau} F^{\sigma \tau} \\
\vartheta_{\mu}^{2}= & \frac{1}{8} D^{\sigma} F_{\sigma \mu} \\
\vartheta_{\mu}^{3}= & -\frac{1}{16} \varepsilon_{\mu \sigma \tau} D^{\sigma} D_{\rho} F^{\rho \tau}+\frac{g}{48} \varepsilon_{\mu \sigma \tau}\left[F^{\sigma \rho}, F_{\rho}^{\tau}\right] \\
\vartheta_{\mu}^{4}= & -\frac{5}{128} D^{2} D^{\rho} F_{\rho \mu}+\frac{5}{128} D^{\nu} D_{\mu} D^{\lambda} F_{\lambda \nu} \\
& -\frac{7}{192} g\left[D^{\rho} F_{\rho \tau}, F_{\mu}^{\tau}\right]-\frac{g}{48}\left[D_{\nu} F_{\mu \lambda}, F^{\lambda \nu}\right] .
\end{aligned}
$$

The equation (雨) expresses the classical equivalence, up to a field redefinition, between the topological massive Yang-Mills and the pure Chern-Simons action.

It seems therefore natural to ask ourselves if, due to the equation (4), the vector supersymmetry may still be present when the Yang-Mills action is added to the pure topological Chern-Simons term. This is the purpose of the present letter. We shall be able, in particular, to prove that the topological massive Yang-Mills action (1) does posses in fact the vector supersymmetry. Furthermore, unlike the pure Chern-Simons case, the vector supersymmetry is now realized nonlinearly on the fields and, in analogy with the field redefinition (5), can be cast in the form of a power series in $1 / \mathrm{m}$.

The work is organized as follows. In Sect.2 we briefly recall the supersymmetric structure of pure Chern-Simons. The Sect.3 is devoted to the analysis of the vector susy in the case of the topological massive Yang-Mills theory. 


\section{Vector susy in the pure Chern-Simons the- ory}

In order to recall the main features of the vector susy in pure Chern-Simons theory [6, 7], we first quantize the model by adopting a transverse Landau gauge condition. For the gauge fixed action we get

$$
\Sigma_{C S}(A)=S_{C S}(A)+\operatorname{tr} \int d^{3} x\left(b \partial^{\mu} A_{\mu}+\bar{c} \partial^{\mu} D_{\mu} c\right),
$$

where the fields $b, c, \bar{c}$ denote respectively that Lagrange multiplier and the Faddeev-Popov ghosts. For the BRST transformations we have

$$
\begin{aligned}
s A_{\mu} & =-D_{\mu} c=-\left(\partial_{\mu} c+g\left[A_{\mu}, c\right]\right) \\
s c & =g c^{2} \\
s \bar{c} & =b \\
s b & =0
\end{aligned}
$$

with

$$
s \Sigma_{C S}=0 .
$$

It is easily checked now that the quantized action $\Sigma_{C S}$ is left invariant by the following vector type transformations:

$$
\begin{aligned}
\delta_{\mu} c & =A_{\mu}, \\
\delta_{\mu} A_{v} & =\varepsilon_{\mu v \rho} \partial^{\rho} \bar{c}, \\
\delta_{\mu} b & =\partial_{\mu} \bar{c}, \\
\delta_{\mu} \bar{c} & =0,
\end{aligned}
$$

with

$$
\delta_{\mu} \Sigma_{C S}=\operatorname{tr} \int d^{3} x\left(A_{\mu} \frac{\delta}{\delta c}+\varepsilon_{\mu v \rho} \partial^{\rho} \bar{c} \frac{\delta}{\delta A_{v}}+\partial_{\mu} \bar{c} \frac{\delta}{\delta b}\right) \Sigma_{C S}=0 .
$$

In addition, the generators $\delta_{\mu}, s$ give rise to the following anti-commutation relations 


$$
\begin{aligned}
\left\{\delta_{\mu}, \delta_{v}\right\} & =0 \\
\left\{s, \delta_{\mu}\right\} & =\partial_{\mu}+(\text { equations of motion) },
\end{aligned}
$$

which, closing on-shell on the translations, yield a Wess-Zumino type supersymmetric algebra. The eqs.(10) are known as the vector susy transformations, since the generator $\delta_{\mu}$ carries a Lorentz index. It is worth noticing here that the vector susy (10) is linearly realized on the fields and that it has been proven to play an important role on the proof of the ultraviolet finiteness of the Chern-Simons theory [3, [0].

\section{The case of topological massive Yang-Mills}

In order to discuss the existence of the vector susy for the topological massive Yang-Mills action (1), we remind that the coefficients $\vartheta_{\mu}^{n}$ in the equations (5), (6) have been proven [9] to transform covariantly under the BRST transformations, i.e.

$$
s \vartheta_{\mu}^{n}=g\left[\vartheta_{\mu}^{n}, c\right] .
$$

As a consequence, the redefined field $\widehat{A}_{\mu}$ transforms as a connection, namely

$$
s \widehat{A}_{\mu}=-\left(\partial_{\mu} c+g\left[\widehat{A}_{\mu}, c\right]\right) .
$$

Therefore, if we choose as the gauge fixing condition a Landau condition for the connection $\widehat{A}_{\mu}, i$. $e$.

$$
\partial^{\mu} \widehat{A}_{\mu}=0
$$

for the quantized topological massive Yang-Mills action we get:

$$
\begin{aligned}
\Sigma_{T M Y M}(A)= & S_{T M Y M}(A) \\
& +\operatorname{tr} \int d^{3} x\left(b \partial^{\mu} \widehat{A}_{\mu}+\bar{c} \partial^{\mu}\left(\partial_{\mu} c+g\left[\widehat{A}_{\mu}, c\right]\right)\right) .
\end{aligned}
$$

Moreover, recalling from eq. (4) that

$$
S_{T M Y M}(A)=S_{C S}(A)+\frac{1}{4 m} \operatorname{tr} \int d^{3} x F^{2}=S_{C S}(\widehat{A}),
$$


the equation (16) becomes

$$
\begin{aligned}
\Sigma_{T M Y M}(A) & =\Sigma_{C S}(\widehat{A}) \\
& =S_{T M Y M}(\widehat{A})+\operatorname{tr} \int d^{3} x\left\{b \partial^{\mu} \widehat{A}_{\mu}+\bar{c} \partial^{\mu}\left(\partial_{\mu} c+g\left[\widehat{A}_{\mu}, c\right]\right)\right\}
\end{aligned}
$$

The expression (18) is easily recognized to be the quantized Chern-Simons action (7) viewed as a functional of the gauge connection $\widehat{A}_{\mu}$ instead of $A_{\mu}$. It is apparent therefore that the quantized action $\Sigma_{T M Y M}(A)$ is left invariant by the following vector transformations

$$
\operatorname{tr} \int d^{3} x\left(\widehat{A}_{\mu} \frac{\delta}{\delta c}+\varepsilon_{\mu v \rho} \partial^{\rho} \bar{c} \frac{\delta}{\delta \widehat{A}_{v}}+\partial_{\mu} \bar{c} \frac{\delta}{\delta b}\right) \Sigma_{T M Y M}(A)=0
$$

Moving now from the connection $\widehat{A}_{\mu}$ to the gauge field $A_{\mu}$, we obtain the nonlinear vector susy Ward identity for the quantized topological massive Yang-Mills action we were looking for

$$
\begin{gathered}
\mathcal{W}_{\mu} \Sigma_{T M Y M}=0, \\
\mathcal{W}_{\mu}=\operatorname{tr} \int d^{3} x\left(\left(A_{\mu}+\sum_{n=1}^{\infty} \frac{1}{m^{n}} \vartheta_{\mu}^{n}\right) \frac{\delta}{\delta c}+\varepsilon_{\mu \alpha \beta} \partial^{\alpha} \bar{c} \mathcal{M}_{\lambda}^{\beta}(x) \frac{\delta}{\delta A_{\lambda}}+\partial_{\mu} \bar{c} \frac{\delta}{\delta b}\right),
\end{gathered}
$$

where the kernel $\mathcal{M}_{\lambda}^{\beta}(x)$ is given by

$$
\mathcal{M}_{\lambda}^{\beta}(x)=\int d^{3} y \frac{\delta A_{\lambda}(y)}{\delta \widehat{A}_{\beta}(x)},
$$

and is easily obtained by inverting the transformation (5). We see thus that, as already mentioned, the vector susy Ward identity for the massive topological Yang-Mills theory is realized nonlinearly, due to the presence of the coefficients $\vartheta_{\mu}^{n}$ and of the kernel $\mathcal{M}_{\lambda}^{\beta}(x)$ in the Ward operator $\mathcal{W}_{\mu}$. Furthermore, as in the case of the field redefinition (5), the vector susy Ward operator $\mathcal{W}_{\mu}$ can be expanded in a power series in $1 / m$, yielding

$$
\mathcal{W}_{\mu}=\sum_{n=0}^{\infty} \frac{1}{m^{n}} \mathcal{W}_{\mu}^{n} .
$$

For the first terms of the series we get

$\mathcal{W}_{\mu}^{0}=\operatorname{tr} \int\left(A_{\mu} \frac{\delta}{\delta c}+\varepsilon_{\mu \alpha \beta} \partial^{\beta} \bar{c} \frac{\delta}{\delta A_{\alpha}}+\partial_{\mu} \bar{c} \frac{\delta}{\delta b}\right)$ 


$$
\begin{aligned}
\mathcal{W}_{\mu}^{1}= & \operatorname{tr} \int\left(\vartheta_{\mu}^{1} \frac{\delta}{\delta c}+\frac{1}{2} D_{\rho} \partial^{\rho} \bar{c} \frac{\delta}{\delta A^{\mu}}-\frac{1}{2} D_{\mu} \partial^{\rho} \bar{c} \frac{\delta}{\delta A^{\rho}}\right) \\
\mathcal{W}_{\mu}^{2}= & \operatorname{tr} \int\left(\vartheta_{\mu}^{2} \frac{\delta}{\delta c}+\frac{1}{8} \varepsilon_{\mu \nu \rho}\left(3 D^{2} \partial^{\rho} \bar{c} \delta^{\nu \alpha}-3 D^{\nu} D^{\alpha} \partial^{\rho} \bar{c}-\left[\partial^{\rho} \bar{c}, F^{\nu \alpha}\right]\right) \frac{\delta}{\delta A^{\alpha}}\right) \\
\mathcal{W}_{\mu}^{3}= & \operatorname{tr} \int\left(\vartheta_{\mu}^{3} \frac{\delta}{\delta c}+\frac{1}{2}\left(\frac{1}{4} D_{\nu}\left[\partial^{\rho} \bar{c}, F_{\rho}^{\nu}\right]+\frac{5}{8} D^{2} D_{\rho} \partial^{\rho} \bar{c}-\left[\partial^{\rho} \bar{c}, \vartheta_{\rho}^{2}\right]\right) \frac{\delta}{\delta A^{\mu}}\right. \\
& +\frac{1}{2}\left(\frac{3}{8}\left[\partial^{\nu} \bar{c}, D^{\rho} F_{\nu \mu}\right]-\left[\partial^{\rho} \bar{c}, \vartheta_{\mu}^{2}\right]-\frac{9}{8} D_{\mu}\left[\partial^{\nu} \bar{c}, F_{\nu}^{\rho}\right]\right. \\
& -\frac{9}{8} D_{\nu}\left[\partial^{\nu} \bar{c}, F_{\mu}{ }^{\rho}\right]-\frac{5}{4} D^{\rho}\left[\partial^{\nu} \bar{c}, F_{\nu \mu}\right]+\frac{1}{4} D^{\nu}\left[\partial^{\rho} \bar{c}, F_{\mu \nu}\right] \\
& \left.\left.+\frac{5}{8}\left(D_{\nu} D^{\rho} D_{\mu} \partial^{\nu} \bar{c}-D^{2} D_{\mu} \partial^{\rho} \bar{c}-D_{\mu} D^{\rho} D_{\nu} \partial^{\nu} \bar{c}\right)\right) \frac{\delta}{\delta A^{\rho}}\right) .
\end{aligned}
$$

Notice also that $\mathcal{W}_{\mu}^{0}$ in the eqs.(23) coincides with the vector susy Ward operator $\delta_{\mu}$ (11) for the pure Chern-Simons. Finally, according to eqs. (12), for the algebra generated by the BRST operator and by the vector susy Ward operator $\mathcal{W}_{\mu}$ we obtain the following on-shell Wess-Zumino type algebra

$$
\begin{aligned}
\left\{s, \mathcal{W}_{\mu}\right\} A_{v} & =\partial_{\mu} A_{v}+2 \varepsilon_{v \mu \rho} \int d^{3} y \frac{\delta A_{\lambda}(y)}{\delta \widehat{A}_{\rho}(x)} \frac{\delta \Sigma_{T M Y M}(A)}{\left.\delta A_{\lambda(} y\right)} \\
\left\{s, \mathcal{W}_{\mu}\right\} c & =\partial_{\mu} c \\
\left\{s, \mathcal{W}_{\mu}\right\} \bar{c} & =\partial_{\mu} \bar{c} \\
\left\{s, \mathcal{W}_{\mu}\right\} b & =\partial_{\mu} b .
\end{aligned}
$$




\section{Acknowledgements}

The Conselho Nacional de Pesquisa e Desenvolvimento (CNPq/Brazil), the Faperj, Fundação de Amparo à Pesquisa do Estado do Rio de Janeiro and the SR2-UERJ are gratefully acknowledged for financial support.

\section{References}

[1] D. Birmingham, M. Blau, M. Rakowski and G. Thompson, Phys. Rep. 209 (1991) 129;

[2] D. Birmingham, M. Rakowski and G. Thompson, Nucl. Phys. B329 (1990) 83;

D. Birmingham and M. Rakowski, Mod. Phys. Lett. A4 (1989) 1753 ;

[3] F. Delduc, F. Gieres and S. P. Sorella, Phys. Lett. B225 (1998) 367;

F. Delduc, C. Lucchesi, O. Piguet and S. P. Sorella, Nucl. Phys. B346 (1990) 313;

[4] E. Guadagnini, N. Maggiore and S. P. Sorella, Phys. Lett. B255 (1991) 65;

N. Maggiore and S. P. Sorella, Int. Journ. Mod. Phys. A8 (1993) 929;

C. Lucchesi, O. Piguet, S. P. Sorella, Nucl. Phys. B395 (1993) 325;

[5] D. Birmingham, M. Rakowski, Phys. Lett. B269 (1991) 103;

D. Birmingham, M. Rakowski, Phys. Lett. B275 (1992) 289;

D. Birmingham, M. Rakowski, Phys. Lett. B289 (1992) 271;

A. Brandhuber, O. Moritsch, M. W. de Oliveira, O. Piguet and M. Schweda, Nucl. Phys. B341 (1994) 173;

[6] O. Piguet and S. P. Sorella, Algebraic Renormalization, Monographs Series, Vol. m28, Springer-Verlag, Berlin 1995; 
[7] C. A. G. Sasaki, S. P. Sorella, O. S. Ventura and L. C. Q. Vilar, Journ. Math. Phys. 39 (1998) 848;

[8] V. E. R. Lemes, C. Linhares de Jesus, C. A. G. Sasaki, S. P. Sorella, O. S. Ventura and L. Q. C. Vilar, Phys. Lett. B418 (1998) 324;

[9] V.E.R. Lemes, C. Linhares de Jesus, S.P. Sorella, L.C.Q. Vilar and O.S. Ventura, Chern-Simons as a geometrical set up for three dimensional gauge theories, CBPF-NF-078/97, hep-th/9801021, to appear in Phys. Rev. D;

[10] S. Deser, R. Jackiw and S. Templeton, Ann. Phys. 140 (1982) 372; 